\title{
Chemo-mechanical modelling of the external sulfate attack in concrete
}

\author{
Nicola Cefis ${ }^{\mathrm{a}}$, Claudia Comi ${ }^{\mathrm{a}, *}$ \\ ${ }^{a}$ Department of Civil and Environmental Engineering, Politecnico di Milano \\ Piazza Leonardo da Vinci, 32, 20133 Milano (Italy)
}

\begin{abstract}
This paper is focused on the modeling of the mechanical consequences of external sulfate attack in concrete structures under partially or fully saturated conditions. To this purpose a weakly coupled approach is developed: first the moisture content is computed through a simplified diffusion model, then a reactive-diffusion model allows for the computation of the expansive products of the reaction occurring between the aluminates of the cement paste and the incoming sulfate ions, finally the solution of a nonlinear mechanical problem gives the expansion, the stress state and the degradation induced by the reaction. The mechanical problem makes use of a multiphase elasto-damage model, developed in this work and accounting for both chemical and mechanical damage. The model is validated by simulating various experimental tests on concrete specimens subject to external sulfate attack and then applied to the simulation of a reduced scale structure of a tunnel lining.
\end{abstract}

Keywords: Concrete, sulfate attack, delayed ettringite formation, damage.

\section{Introduction}

The sulfate attack in concrete consists of a complex set of reactions between sulfate ions (coming from the external environment or released by the cement paste after high heating) and the hydrate calcium aluminates present in

\footnotetext{
* Corresponding author

Email address: claudia.comi@polimi.it (Claudia Comi)
} 
5 the cement paste. In the first case the phenomenon is called Extrenal Sulfate Attack (ESA), while in the second case the phenomenon is called Internal Sulfate Attack (ISA) or Delayed Ettringite Formation (DEF). In both cases one of the final reaction products is the secondary ettringite that, forming within the hardened matrix, can generate swelling and microcracks formation inside the material. The mechanical effects on the structural elements can be very serious up to compromise the reliability of the entire construction ([1], [2]). When a concrete structure is located in a geological site naturally rich in sulfates the phenomenon can not be avoided and one can only try to mitigate the mechanical consequences of the phenomenon of reducing the permeability, by using 15 low-aluminates cements or by isolating the external surfaces.

In the literature there are many works focused on the description of the chemical mechanisms and of the mechanical consequences of sulfate attack. Although there is not unanimous agreement on the mechanisms that cause the expansion of the material [3], many authors agree on attributing it to the formation of secondary ettringite ([4], [5], [6], [7], [8]). The rate of reactions and the consequent mechanical response of material depends of environmental factors (species and concentration of sulfate, $\mathrm{pH}$ of the solution, humidity, temperature) and intrinsic material properties (w/c ratio, chemical composition of the cement paste, pore distribution, diffusivity properties). Many experimental campaigns have been conducted on mortar and concrete to charaterize specific features of the phenomenon, see e.g. [9], [10], [11], [12], [13].

In ESA the sulfates penetrate within the porous network of the material which can be initially in partially saturated conditions. The variation in time of the moisture content influences the diffusion of sulfates. The computation of the 30 amount of the reaction expansive products therefore requires a proper diffusionreaction model, accounting for the variation of the degree of saturation, while a mechanical model is required for the prediction of swelling and material damage.

The mechanical model can be formulated in different ways. In [5] the volumetric expansion is treated as an eigenstrain and the mechanical response is 35 modeled by a simple uniaxial stress-strain law. In [6] a micromechanical model 
based on the Eshelby solution of the equivalent inclusion method is implemented to determine the eigenstrain generated by the formation of ettringite crystals in cement paste. In [14] the expansion is related to the crystallization pressure, generated by crystals growing in the pores of the hardened paste. In [15] con-

40 crete affected by ESA is modeled at the meso-scale as a two phase composite constituted by aggregates and reactive cement matrix and the degradation is described by cohesive-crack interface elements. Most of the proposed models does not take into account the variation of moisture conditions. Very seldom these models are applied at structural level.

The present work aims to develop a reliable approach to be used in structural analyses to simulate the mechanical effects of ESA, also accounting for partially saturated conditions. To this purpose the moisture content is computed through a simplified diffusion model, [16]. The reactive-diffusion model proposed in [5] and further developed in [15], modified to account for partially saturated conditions, is then used to compute the sulfate molar concentration and the amount of formed ettringite. The ettringite formation implies a volume increase and, once the initial porosity is filled, it induces a volumetric deformation. In the context of the Biot's theory of porous media, and similarly to what proposed in [17], [18], [19] in the case of alkali silica reaction, the concrete subject to sulfate attack is represented as a multi-phase medium. Two phenomenological isotropic damage variables describe the chemical degradation and the stress-induced degradation. The model has been validated by simulating the experimental tests in isothermal conditions presented in [13] and [20], and then used to simulate the behaviour of a reduced-scale model of a tunnel 60 lining reported in [1].

\section{Chemical framework and mechanical consequences}

The sulfate attack of concrete manifests itself through a set of topochemical reactions occurring between the hydrated products of cement and the sulfate. In the most common case the sulfates anions come from the ionic dissociation of 
sodium sulfate through reactions with the portlandite $C H$ or the gel $C-S-H$ leading to the formation of gypsum $C \bar{S} H_{2}$ ([21], [4], [5], [15]). The initial process can be defined by the reactions:

$$
\begin{array}{r}
C H+\mathrm{Na}_{2} \mathrm{SO}_{4}+H \rightarrow C \bar{S} H_{2}+2 \mathrm{NaOH} \\
C-S-H+\mathrm{Na}_{2} \mathrm{SO}_{4}+H \rightarrow C \bar{S} H_{2}+2 \mathrm{Na}^{++}
\end{array}
$$

Then, the reactions between the gypsum and the hydrated and unreacted aluminates lead to the formation of ettringite $C_{6} A \bar{S}_{3} H_{32}$ :

$$
\begin{gathered}
C_{4} A \bar{S} H_{12}+2 C \bar{S} H_{2}+16 H \rightarrow C_{6} A \bar{S}_{3} H_{32} \\
C_{4} A H_{13}+2 C H+3 \bar{S}+17 H \rightarrow C_{6} A \bar{S}_{3} H_{32} \\
3 C_{4} A F+12 C \bar{S} H_{2}+a H \rightarrow 4\left(C_{6} A \bar{S}_{3} H_{32}\right)+2\left[(A, F) H_{3}\right] \\
C_{3} A+3 C \bar{S} H_{2}+26 H \rightarrow C_{6} A \bar{S}_{3} H_{32}
\end{gathered}
$$

In reactions (1)-(6) the italic letters refer to the cement notation defined in table 651.

The amount of secondary ettringite formed through reactions (1)-(6) depends on the composition of the cement and in particular on the amount of tricalcium aluminate initially present. Several experimental campaigns [11], [10] have shown that cements with lower content of $C_{3} A$ are significantly less 70 affected by the delayed ettringite formation.

The sulfate attack causes the overall expansion of concrete and the formation of microcracks with progressive reduction of strength and stiffness and possible macro-cracking and spalling of the material.

This phenomenon is mainly due to two interacting effects. The first effect is the dissolution of calcium hydroxide and gel $C-S-H$ caused by the reaction with the sulfates. The gel $C-S-H$ is the main responsible of strength and stiffness in the hardened concrete and its dissolution with calcium leaching causes 
Principal raw materials

Water

Sulfur trioxide

Calcium oxide

Aluminium oxide

Silicon dioxide

Iron oxide

\section{Clinker components}

Tricalcium silicate

Dicalcium silicate

Tricalcium aluminate

Tetracalcium alumino ferrite
Chemical formula

$\mathrm{H}_{2} \mathrm{O}$

$\mathrm{SO}_{3}$

$\mathrm{CaO}$

$\mathrm{Al}_{2} \mathrm{O}_{3}$

$\mathrm{SiO}_{2}$

$\mathrm{Fe}_{2} \mathrm{O}_{3}$

Chemical formula

$(\mathrm{CaO})_{3} \mathrm{SiO}_{2}$

$(\mathrm{CaO})_{2} \mathrm{SiO}_{2}$

$(\mathrm{CaO})_{3} \mathrm{Al}_{2} \mathrm{O}_{3}$

$4 \mathrm{CaO} \cdot \mathrm{Al}_{2} \mathrm{O}_{3} \cdot \mathrm{Fe}_{2} \mathrm{O}_{3}$
Cement notation

H

$\bar{S}$

C

A

$S$

F

Table 1: Raw materials and clinker components: chemical formula and cement notation

micro-cracks formation. A similar phenomenon is the decalcification of calcium hydroxide by the interstitial solution poor in calcium. The calcium hydroxide does not contribute to the mechanical strength of concrete but its consumption causes an increase in porosity facilitating the migration of aggressive species inside the material. The second main phenomenon is the formation of expansive ettringite in the pores. This phenomenon is more relevant in ESA where the penetration of sulfate, and the resulting reaction, takes place progressively: the outer layers react before and tend to expand. In this region the solid skeleton is subject to a prevailing compression state. The inner layers instead, in which the reaction has not yet occurred, constrain the expansion of the outer layers and are therefore subject to tensile stress which can exceed the material strength and cause cracks formation.

Leaching and expansion due to the ettringite formation are two phenomena strongly interacting. Microscopic observations on mortar samples subject to a combination of leaching and ettringite formation, [22], show that three distinct 


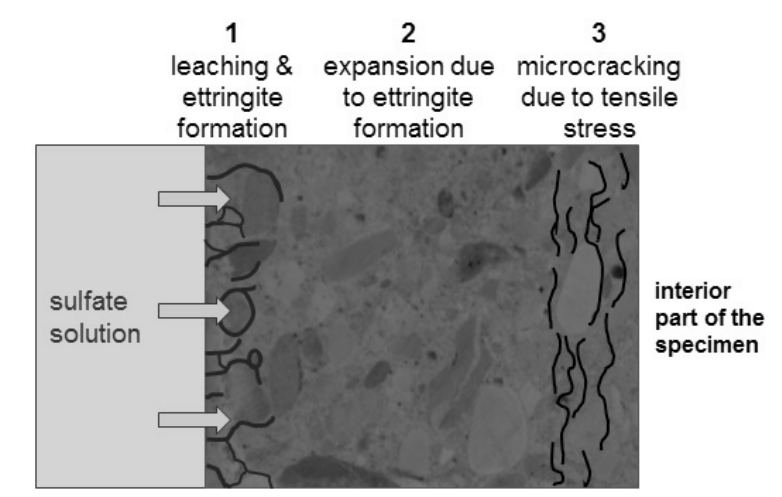

Figure 1: Schematic view of the degradation and formation of three zones in a specimen affected by ESA

zones can be identified, as schematically sketched in figure 1. The first zone is characterized by diffuse microcracking due to leaching and by ettringite formation. In the second zone there is no leaching and no visible damage is present, but there is delayed ettringite formation. This region tends to expand with respect to the inner part and it is therefore subject to compressive stress. In the third zone the reaction is not yet developed and the material is cracked due to tensile stress arising for compatibility of deformation with the outer reactive layer.

\section{Chemo-elastic damage model in fully saturated conditions}

\subsection{Porous medium}

Within the theory of porous materials [23], at the mesoscale the reacting material can be represented by a superposition of two phases: the homogenized concrete skeleton (including the nonreactive material $(u)$ and the reactive part of solid matrix $(c)$ )and the homogenized fluid (including water $(w)$, sulfates $(s)$ and reaction products $(e)$ ). This approach, used for the study of other chemical reactions in concrete $([24],[17])$, considers the material in conditions of total saturation. In the case of SA the sulfates reacts with the aluminates of the cement paste and lead to the formation of ettringite crystals. The stress exerted 
by these crystals is assumed to be isotropic, so that the secondary ettringite is considered as a fluid-like constituent exerting a pressure on the solid skeleton. A schematic representation of the porous material particle as superposition of skeleton and fluid particles is shown in figure 2. The total volume $V$ of the representative volume element RVE at each instant is the sum of the volume of the solid matrix $V_{s}$ and the volume of the fluid inside the pores $V_{f}$. The lagrangian porosity $\phi$ is defined as the ratio between the volume currently filled by the fluid and the initial total volume of RVE $V_{0}$

$$
\phi=\frac{V_{f}}{V_{0}}
$$

The kinetic of the porous material is described by two variables: the strain tensor $\boldsymbol{\epsilon}$ of the skeleton and the variation of fluid content $\zeta$. The latter is defined as

$$
\zeta=\frac{m_{f}-m_{f 0}}{\rho_{f}}
$$

where $m_{f}$ and $m_{f 0}$ are the fluid mass contents at the current and initial state and $\rho_{f}$ is the fluid intrinsic mass density.
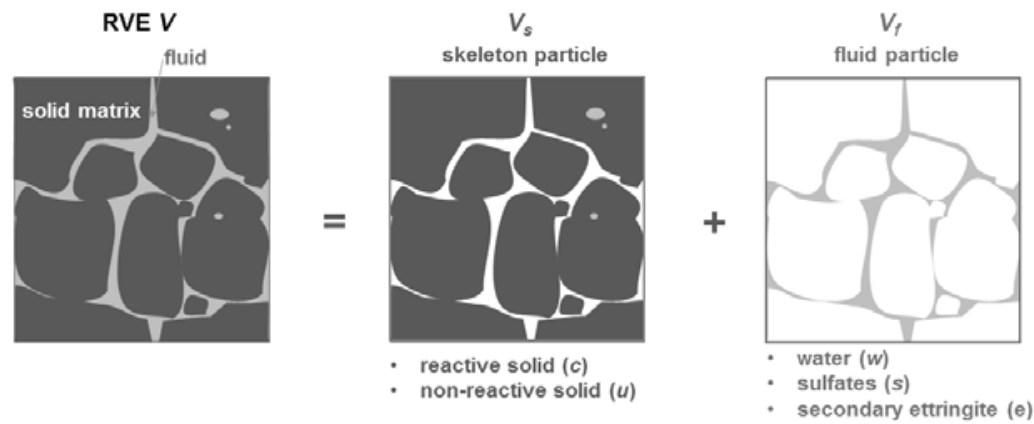

Figure 2: Biphase model of concrete affected by sulfate sttack 


\subsection{Compatibility and equilibrium equations}

The kinematic model adopted is based on the assumption of small strains and displacements. The compatibility conditions read

$$
\begin{array}{ll}
\boldsymbol{\epsilon}=\frac{1}{2}\left(\operatorname{grad} \mathbf{u}+\operatorname{grad}^{T} \mathbf{u}\right) & \text { in } \Omega \\
\mathbf{u}=\overline{\mathbf{u}} & \text { on } \Gamma_{u}
\end{array}
$$

where $\mathbf{u}$ is the displacement vector, $\Omega$ is the volume of the body and $\overline{\mathbf{u}}$ are the displacements on the constrained boundary $\Gamma_{u}$ of the body.

Neglecting dynamic effects, the equilibrium equations are

$$
\begin{array}{ll}
\operatorname{div} \boldsymbol{\sigma}+\rho \mathbf{b}=\mathbf{0} & \text { in } \Omega \\
\boldsymbol{\sigma} \mathbf{n}=\mathbf{f} & \text { on } \Gamma_{\sigma}
\end{array}
$$

where $\boldsymbol{\sigma}$ is the stress tensor, $\rho \mathbf{b}$ is the body force of the mixture (solid and fluid), $\mathbf{f}$ is the external force at the free boundary $\Gamma_{\sigma}$.

\subsection{Balance of mass}

Under the hypothesis of infinitesimal transformation, the mass balance can be expressed for each constituent $\alpha$ in the form:

$$
\frac{d m_{\alpha}}{d t}=-\operatorname{div}\left(\rho_{\alpha} \mathbf{M}_{\alpha}\right)+\stackrel{\circ}{m}_{\rightarrow \alpha}
$$

where $\rho_{\alpha}$ and $m_{\alpha}=\rho_{\alpha} \phi_{\alpha}$ are, respectively, the intrinsic density and the mass content of constituent $\alpha, \mathbf{M}_{\alpha}$ is the flux of the constituent $\alpha$ with respect to the

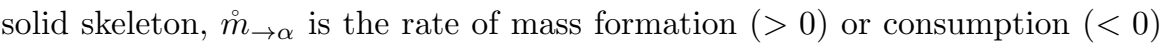
of component $\alpha$.

The mass concentration $m_{\alpha}$ is directly related to the molar concentration $n_{\alpha}$ through the molar mass $\mathcal{M}_{\alpha}$

$$
m_{\alpha}=n_{\alpha} \mathcal{M}_{\alpha}
$$

In diffusion-reaction problems the equations of mass balance (11) are more conveniently expressed in terms of molar concentrations:

$$
\frac{d n_{\alpha}}{d t}=-\operatorname{div}\left(\mathbf{j}_{\alpha}\right)+\stackrel{\circ}{n} \rightarrow \alpha
$$


where $\mathbf{j}_{\alpha}$ is the flow of moles of component $\alpha$

$$
\mathbf{j}_{\alpha}=\frac{\rho_{\alpha}}{\mathcal{M}_{\alpha}} \mathbf{M}_{\alpha}
$$

In a fully saturated porous medium, under isothermal conditions, without electrochemical coupling phenomena, the flow term of the fluid components can be modeled through the Fick's law. With this hypothesis, the diffusion is driven by the concentration gradient only

$$
\mathbf{j}_{\alpha}=-D_{\alpha} \operatorname{grad}\left(n_{\alpha}\right)
$$

The equations of balance (13), written for each constituent of the concrete affected by SA, read

$$
\begin{aligned}
u: & \frac{d n_{u}}{d t}=0 \\
c: & \frac{d n_{c}}{d t}=\stackrel{\circ}{n}_{\rightarrow c} \\
w: & \frac{d n_{w}}{d t}=-\operatorname{div}\left(\mathbf{j}_{w}\right)+\stackrel{\circ}{n} \rightarrow w \\
s: & \frac{d n_{s}}{d t}=-\operatorname{div}\left(\mathbf{j}_{s}\right)+\stackrel{\circ}{n} \rightarrow s \\
e: & \frac{d n_{e}}{d t}=-\operatorname{div}\left(\mathbf{j}_{e}\right)+\stackrel{\circ}{n} \rightarrow e
\end{aligned}
$$

Note that the solid components, reactive $c$ and non-reactive $u$, have zero relative velocity with respect to the skeleton, therefore in the balance equations the associated flow terms disappear: $\mathbf{j}_{u}=\mathbf{0}, \mathbf{j}_{c}=\mathbf{0}$. Furthermore by definition, for

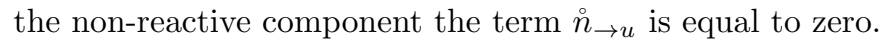

To identify the other terms involved in the molar balances the chemical problem, described by the equations (1)-(6), must be analyzed. The first set of equations (1)-(2) describes the reaction between sulfate anions and hydration products of cement paste (silicates and portandite) with consequent formation of gypsum. For each mole of sulfate reacted one mole of gypsum is formed. The second set of equations (3)-(6) expresses the reactions of gypsum with the calcium aluminates to form ettringite. In the following we consider that the sulfate, spreading in the material, completely reacts forming gypsum, hence the 
variations of the molar concentration of sulfates and gyspum are equal and can be obtained by equation (19).

In the process of ettringite formation, the reactive part of the solid matrix $(c)$ is represented by the calcium aluminates. For this reason, equation (17) is used to model the depletion of aluminates. Following the approach proposed by [5] and [15] equations (3)-(6) are expressed in the lumped form

$$
C_{e q}+q \bar{S}+r H \rightarrow C_{6} A \bar{S}_{3} H_{32}
$$

where $C_{e q}$ is the equivalent grouping of calcium aluminates

$$
C_{e q}=\sum_{i=1}^{4} \gamma_{i} P_{i} \quad \gamma_{i}=\frac{n_{c i}}{\sum_{i=1}^{4} n_{c i}}
$$

$n_{c i}$ is the molar concentration of the single species of calcium aluminate $P_{i}$ ( $P_{1}=C_{4} A \bar{S} H_{12}$ mono-sulphoaluminate, $P_{2}=C_{4} A H_{13}$ tetra-hydrated aluminate, $P_{3}=C_{4} A F$ alumino-ferrite and $P_{4}=C_{3} A$ unreacted tricalcium aluminate), $q=2 \gamma_{1}+3 \gamma_{2}+4 \gamma_{3}+3 \gamma_{4}$ is the stoichiometric weighting coefficient of the sulfate phase and $r$ is the stoichiometric weighting coefficient of water.

During the process the moles of calcium, sulfate and water are transformed in ettringite. The terms of reaction present in equations (17), (18) and (19) are therefore only due to the consumption required for ettringite formation

$$
\begin{aligned}
& \stackrel{\circ}{n \rightarrow c}=\stackrel{\circ}{n}_{e \rightarrow c} \\
& \stackrel{\circ}{n \rightarrow s}=\stackrel{\circ}{n}_{e \rightarrow s} \\
& \stackrel{\circ}{n \rightarrow w}=\stackrel{\circ}{n}_{e \rightarrow w}
\end{aligned}
$$

where $\stackrel{\circ}{\alpha \rightarrow \beta}_{\alpha}=-\grave{n}_{\beta \rightarrow \alpha}$ denotes the number of moles of the $\alpha$-component transformed into the $\beta$-component. Since the diffusion of water inside the porous medium is much more rapid than the reaction, it can be assumed that the reacted moles of water are immediately replaced by the moles coming from the external environment and hence the water content remains constant, and equation (18) needs not to be solved. 
Using (15), (23) and (24) equations (17) and (19) can be written in the form

$$
\begin{aligned}
c: & \frac{d n_{c}}{d t} & =\stackrel{\circ}{n}_{e \rightarrow c} \\
s: & \frac{d n_{s}}{d t} & =\operatorname{div}\left(D_{s} \operatorname{grad}\left(n_{s}\right)\right)+\stackrel{\circ}{n}_{e \rightarrow s}
\end{aligned}
$$

Assuming a second order reaction scheme the reaction term in (27) is expressed as

$$
\stackrel{\circ}{n}_{e \rightarrow s}=-\stackrel{\circ}{n} \rightarrow e=-k n_{c} n_{s}
$$

and accounting for (22) the reaction term in (26) reads

$$
{\stackrel{\circ}{n_{e \rightarrow c}}}=-\stackrel{\circ}{n}_{c \rightarrow e}=-\frac{k}{q} n_{c} n_{s}
$$

Substituting these values into (26)-(27), we obtain the final form of the diffusion reaction equations

$$
\begin{gathered}
\frac{d n_{c}}{d t}=-\frac{k}{q} n_{c} n_{s} \\
\frac{d n_{s}}{d t}=\operatorname{div}\left(D_{s} \operatorname{grad}\left(n_{s}\right)\right)-k n_{c} n_{s}
\end{gathered}
$$

Remark - Under non-uniform temperature conditions, the mass flux of the $\alpha$-component depends also on the temperature gradient through the thermodiffusion term (Soret effect) and equation (15) should be replaced by

$$
\mathbf{j}_{\alpha}=-D_{\alpha}(T) \operatorname{grad}\left(n_{\alpha}\right)-D_{T}(T) n_{\alpha} \operatorname{grad} T
$$

where $D_{T}$ is the thermal diffusion coefficient.

\subsection{Reaction extent}

Considering the reaction of ettringite formation in the lumped form (21), the rates of the moles of reactants and reaction products can be expressed in terms of a single rate of reaction $\dot{\xi}$ as:

$$
\dot{\xi}=-\stackrel{\circ}{n} \rightarrow c=-\frac{\stackrel{\circ}{n}_{\rightarrow s}}{q}=-\frac{\stackrel{\circ}{n}_{\rightarrow w}}{r}=\stackrel{\circ}{n}_{\rightarrow e}
$$


The evolution in time of the reaction can be obtained by integrating (26) from 0 to $t$ and reads

$$
\xi(t)=n_{c}(0)-n_{c}(t)
$$

which represents the molar concentration of the reacted equivalent calcium aluminate. Assuming at time $t=0$ zero molar concentration of secondary ettringite, from (33) it follows that $\xi(t)$ also represents the molar concentration of ettringite at the time $t$. It is also convenient for later use to introduce the reaction extent $\bar{\xi}$ ranging from 0 to 1 defined by

$$
\bar{\xi}=\frac{\xi}{n_{c}(0)}
$$

\subsection{Chemo-elastic model with chemical and mechanical damage}

The state equations for the bi-phase material are derived from the free energy potential $\Psi$. Its definition is based on the following assumptions:

- the solid phase undergoes small deformations, described by the strain tensor $\boldsymbol{\epsilon}$;

- a single scalar variable $\zeta$ measures the variation of the fluid phase content, including water, sulfates and reaction products;

- the calcium leaching induced by sulfate penetration is modeled by a chemical damage variable $d$;

- the mechanical stress-induced damage is modeled by a mechanical damage variable $D$ which depends on two scalar variables $D_{t}$ and $D_{c}$, one describing damage for prevaling tension and the other for prevailing compression stress states: $D=1-\left(1-D_{t}\right)\left(1-D_{c}\right)$;

- isothermal conditions are assumed;

- no permanent strains are considered. 
The free energy is the sum of the energy of the solid skeleton $\Psi_{s}$ and on the fluid $m_{f} \psi_{f}$ (where $\psi_{f}$ is the specific energy of the fluid $f$ ) and reads

$$
\begin{aligned}
& \Psi(\boldsymbol{\epsilon}, \zeta, D, d)=\Psi_{s}+m_{f} \psi_{f}= \\
& =\frac{1}{2}(1-d)(1-D)\left\{2 G \mathbf{e}: \mathbf{e}+K(\operatorname{tr} \boldsymbol{\epsilon})^{2}+M b^{2}\left(\operatorname{tr} \boldsymbol{\epsilon}-\frac{\zeta}{b}\right)^{2}\right\}+ \\
& +\rho_{f} \zeta \psi_{f}
\end{aligned}
$$

In the above equation e is the deviatoric strain tensor, $G$ and $K$ are the shear and bulk moduli of the homogenized concrete skeleton, $M$ and $b$ are the Biot's modulus and the Biot's coefficient respectively. The internal damage variables describe at the mesoscale the effect of microvoids and microcracks formation (see [25]). The multiplicative coupling of the mechanical and chemical damage was previously assumed e.g. in [26], [27].

The static variables (total stress $\boldsymbol{\sigma}$, chemical potential of the fluid $\mu$, strain energy density release rates associated with the mechanical and the chemical damage respectively $Y_{D}$ and $Y_{d}$ ) are obtained by partial derivation of the free energy with respect to each kinematic variable

$$
\begin{aligned}
\boldsymbol{\sigma} & =\frac{\partial \Psi}{\partial \boldsymbol{\epsilon}}=(1-d)(1-D)\left[2 G \mathbf{e}+K \operatorname{tr} \boldsymbol{\epsilon}+M b^{2}\left(\operatorname{tr} \boldsymbol{\epsilon}-\frac{\zeta}{b}\right) \mathbf{1}\right] \\
\mu & =\frac{1}{\rho_{f}} \frac{\partial \Psi}{\partial \zeta}=-\frac{1}{\rho_{f}}(1-d)(1-D) M b\left(\operatorname{tr} \boldsymbol{\epsilon}-\frac{\zeta}{b}\right)+\psi_{f} \\
Y_{D} & =-\frac{\partial \Psi}{\partial D} \\
Y_{d} & =-\frac{\partial \Psi}{\partial d}
\end{aligned}
$$

The chemical potential $\mu$ is related to the fluid pressure $p$ and the specific free energy by

$$
\mu=\frac{p}{\rho_{f}}+\psi_{f}
$$

Substituting this result into equation (38), the expression of fluid pressure is obtained

$$
p=-(1-d)(1-D) M(b \operatorname{tr} \epsilon-\zeta)
$$


The macroscopic stress can hence be expressed in the final form

$$
\boldsymbol{\sigma}=(1-d)(1-D)[2 G \mathbf{e}+K \operatorname{tr} \boldsymbol{\epsilon} \mathbf{1}]-b p \mathbf{1}
$$
the phase which fills the pores, including water and reaction products. Note that, for the sake of simplicity, no-unilateral effect is considered in the model, howevere it can be easily indroduced as in [28]. Furthermore the damage is assumed to be isotropic, described by scalar variables. The anisotropy of the microcrack pattern is recovered at the macro-scale as a structural effect. Possible inclusion of tensorial damage variables has been discussed e.g. in [29].

\subsection{Evolution laws}

The variation of fluid content $\zeta$ can be directly related to the reaction extent through a material parameter $\alpha$

$$
\zeta=\alpha \bar{\xi}
$$

We adopt for $\alpha$ the expression proposed in [5] and used by several Authors [15], [30], [31]:

$$
\alpha=n_{c}(0) \sum_{i=1}^{4} \Delta V_{i} \gamma_{i} \quad \Delta V_{i}=\mathcal{V}_{e}-\mathcal{V}_{i}-\nu_{i} \mathcal{V}_{g y p}
$$

where $\mathcal{V}_{i}, \mathcal{V}_{e}$ e $\mathcal{V}_{\text {gyp }}$ are the molar volumes of each calcium aluminate, of ettringite and of gypsum, $\nu_{i}$ is the stoichiometric coefficient involved in reaction, $\gamma_{i}$ is the ratio between the molar concentration of each aluminate $n_{c, i}$ and the total concentration of aluminates defined in equation (22)b.

The decalcification of material causes the formation of diffuse microcracks which is here described by an isotropic damage variable $d$. Similarly to what proposed in [27], the evolution of chemical damage is expressed as a function of the reaction extent

$$
d=\frac{1-\exp \left(-r_{1} \bar{\xi}\right)}{1+\exp \left(-r_{1} \bar{\xi}+r_{2}\right)} r_{3}
$$

where $r_{1}, r_{2}$ and $r_{3}$ are positive material dependent parameters with $r_{1}>r_{2}$ and $0<r_{3}<1$. As the chemical reaction develops, $\bar{\xi}$ grows and $d$ increases 
from 0 to $r_{3} \frac{1-\exp \left(-r_{1}\right)}{1+\exp \left(-r_{1}+r_{2}\right)}$. The stiffness of the material, and in particular the

Young's modulus, is hence reduced by the factor $(1-d)$.

The mechanical, stress induced, damage of the material is described by two scalar damage variables: $D_{t}$ for damage due to prevailing tensile stress states and $D_{c}$ for damage due to prevailing compression stress states. The evolution of these variables is governed by the loading-unloading conditions proposed in [28] and expressed in term of an inelastic effective stress $\boldsymbol{\sigma}^{\prime \prime}=\boldsymbol{\sigma}+\beta p \mathbf{1}$, with $\beta(\beta \leq b)$ a material parameter which tunes the level of material degradation caused by the fluid pressure and, hence, by the chemical reaction:

$$
f_{i} \leq 0 ; \quad \dot{D}_{i} \geq 0 ; \quad f_{i} \dot{D}_{i}=0 \quad \text { with: } i=t, c
$$

The activation functions $f_{t}$ and $f_{c}$ depend on the first invariant of inelastic effective stress tensor $I_{1}$ and on the second invariant of deviatoric stress tensor $J_{2}$

$$
\begin{aligned}
& f_{t}=J_{2}\left(\boldsymbol{\sigma}^{\prime \prime}\right)-a_{t} I_{1}^{2}\left(\boldsymbol{\sigma}^{\prime \prime}\right)+b_{t} h_{t}\left(D_{t}\right) I_{1}\left(\boldsymbol{\sigma}^{\prime \prime}\right)-k_{t} h_{t}^{2}\left(D_{t}\right) \\
& f_{c}=J_{2}\left(\boldsymbol{\sigma}^{\prime \prime}\right)+a_{c} I_{1}^{2}\left(\boldsymbol{\sigma}^{\prime \prime}\right)+b_{c} h_{c}\left(D_{c}\right) I_{1}\left(\boldsymbol{\sigma}^{\prime \prime}\right)-k_{c} h_{c}^{2}\left(D_{c}\right)
\end{aligned}
$$

where $a_{t}, b_{t}, k_{t}, a_{c}, b_{c}, k_{c}$ are non negative parameters to be identified through experimental tests. The functions $h_{t}\left(D_{t}\right)$ and $h_{c}\left(D_{c}\right)$ govern the hardening and softening behavior

$$
h_{i}\left(D_{i}\right)=\left\{\begin{array}{ll}
1-\left[1-\left(\frac{\sigma_{e i}}{\sigma_{0 i}}\right)\right]\left(1-\frac{D_{i}}{D_{0 i}}\right)^{2} & \text { for } D_{i}<D_{0 i} \\
{\left[1-\left(\frac{D_{i}-D_{0 i}}{1-D_{0 i}}\right)^{c_{i}}\right]^{0.75}} & \text { for } D_{i} \geq D_{0 i}
\end{array} \quad i=t, c\right.
$$

where $\sigma_{e i}$ and $\sigma_{0 i}$ are respectively the elastic limit stress and the peak stress, $D_{0 i}$ is the damage corresponding to the peak stress, the parameters $c_{t}$ and $c_{c}$ govern the softening stress-strain curve. According to the fracture energy regularization technique, in numerical analysis the parameters $c_{i}$ are used to scale the fracture energy density in order to obtain the correct value of the fracture energy with different mesh size.

Remark 1 - Even though the two internal damage variables $d$ and $D$ are intended to phenomenologically describe two different mechanisms of reduction 
of the mechanical properties, the two variables are strongly interacting and their effect on a concrete structure affected by SA is combined. In particular while the chemical damage only develops in the presence of the deleterious chemical reaction due to the sulfate attack, in the present bi-phase model the mechanical damage develops both as a consequence of the external mechanical loads and of the chemical reactions. In fact the reaction extent intervenes in the activation functions $f_{t}$ and $f_{c}$ of the mechanical damage through the inelastic effective stress $\sigma^{\prime \prime}=\sigma+\beta p \mathbf{1}$. When the reaction develops, the pressure in the pores increases and one can have $\boldsymbol{\sigma}^{\prime \prime} \neq \mathbf{0}$ also with zero external loads (stressfree conditions). The strength in tension, shear or compression is therefore dependent on the reaction through $p$. Figure 3 shows the evolution with the pressure of the ratio between the limit tensile strength $\sigma_{t}$ of the material affected by sulfate attack and the nominal tensile strength without chemical reaction $\bar{\sigma}_{t}$ for different values of the material parameter $\beta$

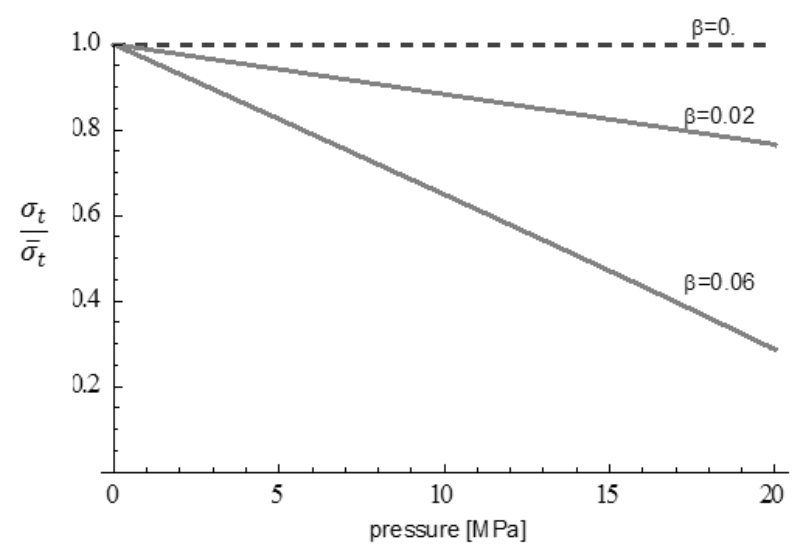

Figure 3: Normalized tensile strength versus pressure exherted by the reaction products

Remark 2 - The present formulation is restricted to isothermal conditions as all tests considered in the paper are carried out at fixed temperature. To account for varying temperature conditions the free energy should be modified to include the temperature field, similarly to what proposed in [17] for the modeling of the alkali-silica reaction. 


\section{Extension to partially saturation conditions}

The model of the previous section is based on the hypotesis that the material be initially saturated with water and that during the ettringite formation the water consumed by the reaction be immediately replaced (locally drained conditions) so that the change in water pressure can be neglected.

Under partially saturated conditions also air and vapour are present in the pores, significant variations in water content can occur in the material and these entail variations of the state of stress and alter the kinetics of the chemical reactions.

\subsection{Equivalent pressure of water}

Let us define the degree of saturation $S_{w}$ as the portion of porosity filled by water $\phi_{w}$ with respect to the total porosity. A change in the water content, or in the degree of saturation, induces a variation of water pressure $p_{w}$. For weakly permeable materials under atmosferic pressure conditions, the pressure of the gas phase $p_{g}$ relative to the atmospheric pressure is zero and the water pressure turns out to be equal to the opposite capillary pressure $p_{c}: p_{w}=-p_{c}$, see [23] for details. The link of the capillary pressure with the degree of saturation can be experimetally obtained in the form of the capillarity curve. In [16] experimental curves are reported and the following interpolation formula is proposed

$$
p_{c}=a\left(S_{w}^{-b}-1\right)^{1-\frac{1}{b}}
$$

with $a$ and $b$ material parameters to be calibrated by fitting the experimental data, see figure 4 .

The equivalent pressure variation due to a variation of the degrees of saturation at the mesoscale for the present damaging model reads

$$
\bar{p}_{w}\left(S_{w}\right)=-(1-d)(1-D) S_{w} \int_{S_{w 0}}^{S_{w}} p_{c} d S_{w}
$$

This contribution adds to the pressure exherted by the delayed ettrigite formation, expressed by eq. (42). 


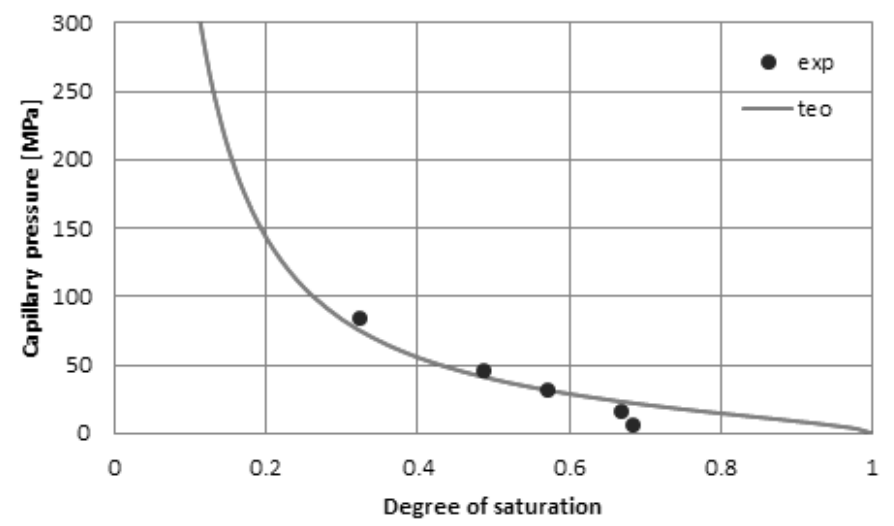

Figure 4: Experimental points and interpolation of the capillary curve reported in [16]

\subsection{Transport of water in liquid form}

In the case of weakly permeable materials, as concrete, it is possible to describe the transport of moisture through a simplified model in which the only variable is the degree of saturation $S_{w}[16]$.

The water diffusion equations is obtained by combining the equation of the mass balance with the Darcy's law and neglecting the contribution of the pressure variation of the gas inside the pores. The nonlinear equation of diffusion is

$$
\phi \frac{\partial S_{w}}{\partial t}+\operatorname{div}\left(D_{w} \operatorname{grad} S_{w}\right)=0
$$

where $\phi$ is the material porosity and $D_{w}$ is the coefficient of diffusion of water. The diffusion coefficient depends on the degree of saturation in the form

$$
D_{w}=\frac{d p_{c}}{d S_{w}} \frac{\kappa}{\eta_{w}} \sqrt{S_{w}}\left(1-\left(1-S_{w}^{b}\right)^{\frac{1}{b}}\right)^{2}
$$

where $\kappa$ is the intrinsic permeability of concrete and $\eta_{w}$ is the dynamic viscosity.

For given initial and boundary conditions on the degree of saturation, the solution of the nonlinear diffusion equation (53) provides the distribution of the degree of saturation, in time and space. In the above diffusion model of water the effect of salts present in the solution is neglected. This assumption 
is justified by the low influence that the salt concentration has on the capillary pressure, see [32].

Generally the boundary conditions are expressed in terms of relative humidity defined as the ratio between the pressure of the vapor and the saturated vapor pressure. The relationship between the degree of saturation and relative humidity $h$ can be formulated by Kelvin's law

$$
p_{c}=-\rho_{w} \frac{R T}{\mathcal{M}_{v}} \ln (h)
$$

where $R=8.3145 \mathrm{JK}^{-1} \mathrm{~mol}^{-1}$ is the universal gas constant, $T$ is the absolute temperature $[K], \mathcal{M}_{v}=18.015 \mathrm{~g} / \mathrm{mol}$ is the molar mass of water vapor and $p_{c}$ is the capillary pressure expressed by the relation (51).

\subsection{Unsaturated diffusion-reaction process}

Let us denote by $n_{s_{L}}=n_{s_{L}}(\mathbf{x}, t)$ the molar concentration of sulfate in the solution, varying in space $\boldsymbol{x}$ and time $t$, and, as in section 3 , by $n_{s}=n_{s}(\mathbf{x}, t)$ the molar concentration of sulfate referred to the unit material volume. In partially saturated media the latter depends on the degree of saturation and the relation between the two concentrations reads

$$
n_{s}(\mathbf{x}, t)=n_{s_{L}}(\mathbf{x}, t) S_{w}(\mathbf{x}, t) \phi
$$

The molar concentration $n_{s_{L}}(\mathbf{x}, t)$ can be computed taking into account the diffusion process of sulfates within the pore solution and the consumption of sulfates due to the ettringite formation, then the problem can be formulated at the representative element volume level through a homogenized technique in terms of the molar concentration $n_{s}$, see [32], [33]. The diffusion of sulfates within the homogenized material is driven by the gradient of concentration and by the fluid movement under the effect of the water content gradient. This second advection term is not present in the fully saturated case. The consumption of sulfates is modeled considering a second order reaction between sulfates and aluminates. The evolution of both the concentration of sulfates and aluminates 
is hence governed by the following equations

$$
\begin{gathered}
\frac{d n_{c}}{d t}=-\frac{k}{q} n_{c} n_{s} \\
\frac{d n_{s}}{d t}-\operatorname{div}\left(\bar{D}_{s} S_{w} \operatorname{grad}\left(\frac{n_{s}}{S_{w}}\right)\right)-D_{w} \frac{n_{s}}{S_{w}} \operatorname{grad} S_{w}=-k n_{c} n_{s}
\end{gathered}
$$

which specialises to equations (30)-(31) in fully saturated conditions $\left(S_{w}=\right.$ 1). In the previous equation $\bar{D}_{s}$ is the coefficient of diffusion of the sulfates depending, for partially saturated media, on the degree of saturation. When the material is unsaturated, the reduction in aqueous phase volume decreases the diffusion properties; as in [32], the following expression is assumed in this work

$$
\bar{D}_{s}=D_{s} S_{w}^{7 / 3}
$$

where $D_{s}$ is the diffusion coefficient for the saturated material.

\section{Numerical analyses}

The chemo-damage constitutive model has been implemented in an ad-hoc finite element code developed in Matlab. For the simulation of ESA on specimens and structures we followed a weakly coupled numerical approach, schematically illustrated in figure 5. A moisture diffusion analysis is first performed to compute the degree of saturation distribution in space and time. Then the chemical reaction-diffusion problem, governed by equations (57)-(58), is solved to compute the evolution of the aluminates content and hence, through equations (35) and (46), the reaction extent $\bar{\xi}$ and the chemical damage $d$. The degree of saturation and the reaction extent are the input of the subsequent mechanical analysis which allows to obtain strain, stress and mechanical damage.

The weakly coupled approach greatly simplifies the numerical solution and is justified by two assumptions which are supported by the experimental results shown in the following section. First, the water consumption due to the reaction of ettringite formation does not alter significantly the degree of saturation, so that the moisture diffusion analysis is independent from the reaction-diffusion 


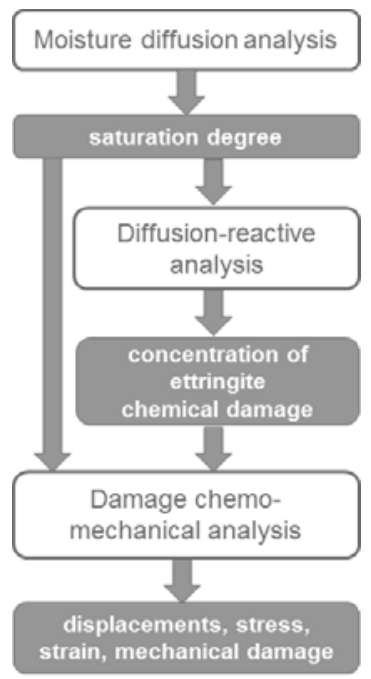

Figure 5: Weakly coupled numerical approach

analysis. Second, the imbibition process is generally very fast when compared to the kinetics of the reaction, thus often the material saturates before the chemically induced damage can develop and alter the permeability.

The above scheme holds under isothermal conditions. If temperature variations are significant, as in the case of exposed real structures, the temperature field should also be preliminary computed through a heat transport analysis governed by the Fourier's equation. The temperature field then enters into the diffusion-reactive analysis in view of eq. (32).

\subsection{ESA on concrete specimens}

The developed model was first validated by simulating the experimental campaign presented in [13] on concrete specimens immersed in solutions with different concetrations of sodium sulfate $\mathrm{Na}_{2} \mathrm{SO}_{4}$. The tests, lasted two years, have been performed on cubes of $15 \mathrm{~cm}$ side and cylinders of diameter $15 \mathrm{~cm}$ and height $30 \mathrm{~cm}$ made with Portland cement CEMII / A-LL 42.5 R. After curing the specimens were immersed in three different solutions (pure water, sodium sulfate solutions at $5 \%$ and $10 \%$ ) and the longitudinal and radial expansion 
were measured at regular intervals. The evolution of the Young modulus was indirectly measured through ultrasonic wave propagation.

The water-diffusion parameter was first calibrated through the data obtained in capillary tests conducted in a climatic chamber at constant temperature and humidity $\left(T=20^{\circ} \mathrm{C}\right.$ and $\left.h=50 \%\right)$. The comparison between the experimental response and the simulation obtained by integration of eq. (53) are reported in figure $6 \mathrm{a}$ in term of the time evolution of the mass variation. Figure $6 \mathrm{~b}$ shows the contour plots of the degree of saturation at different times.

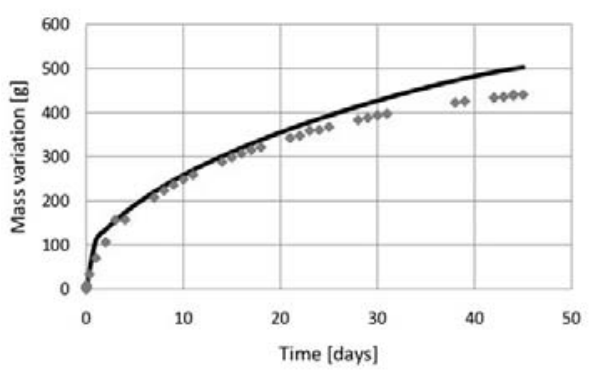

(a)

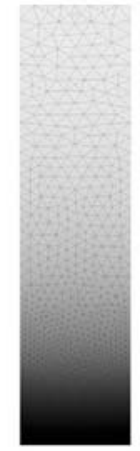

10 days

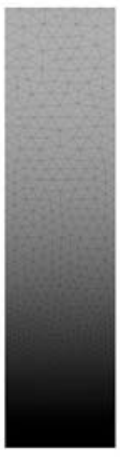

30 days (b)

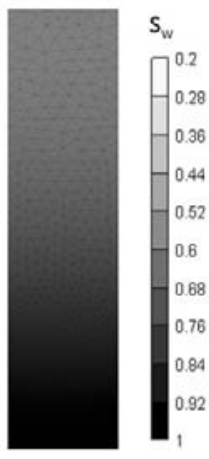

50 days

Figure 6: Capillary test. (a) Mass variation: comparison between experiment and simulation; (b) contour plots of the degree of saturation

The mass variation was also measured for all specimens immersed in different solutions. The results are shown in Fig. 7. It can be observed that the mass uptake due to water imbibition is similar for specimens immersed in sulfate solutions and in pure water and that full saturation is reached after about three months of immersion. These results support the hypothesis of the previous section, thus allowing to neglect the effect of the reaction on the water diffusion. The parameters governing the problem of diffusion-reaction have been calibrated using the profiles of salt penetration on a cube after 400 days of immersion in the $10 \%$ sodium sulfate solution. Figure 8, right part, shows the experimental profile observed on the cross section of the cube after drying. The left part of the same figure shows the contour plot of sulfates numerically obtained using the diffusion 


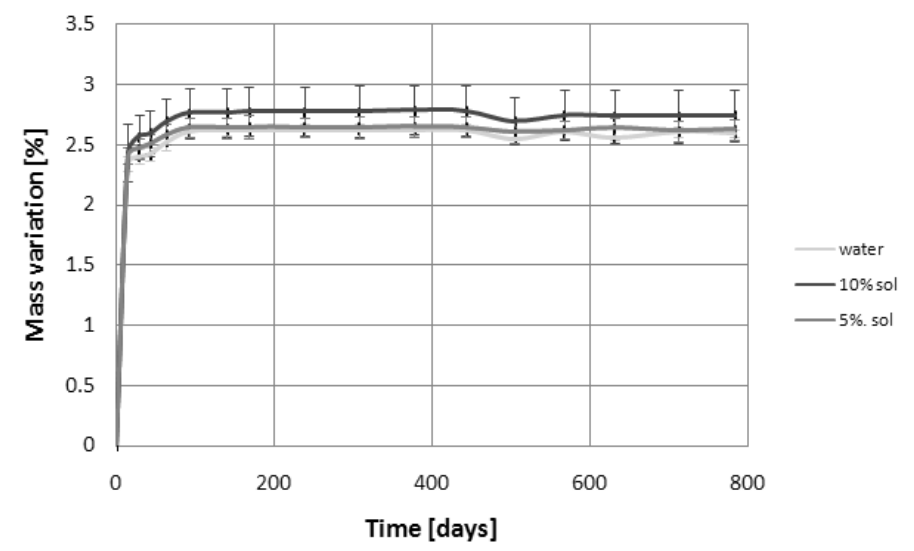

Figure 7: Mass variation of cylinders immersed in pure water and $5 \%$ and $10 \% \mathrm{NaSO}_{4}$ solutions

coefficient for sulfates $D_{S}=7.2 \times 10^{-2} \mathrm{~mm}^{2} /$ days, the reaction coefficient $k=1.12 \times 10^{-4} \mathrm{~m}^{3} /(\mathrm{mol}$ days $)$ and the initial concentration of equivalent aluminate $n_{c}(0)=76 \mathrm{~mol} / \mathrm{m}^{3}$.

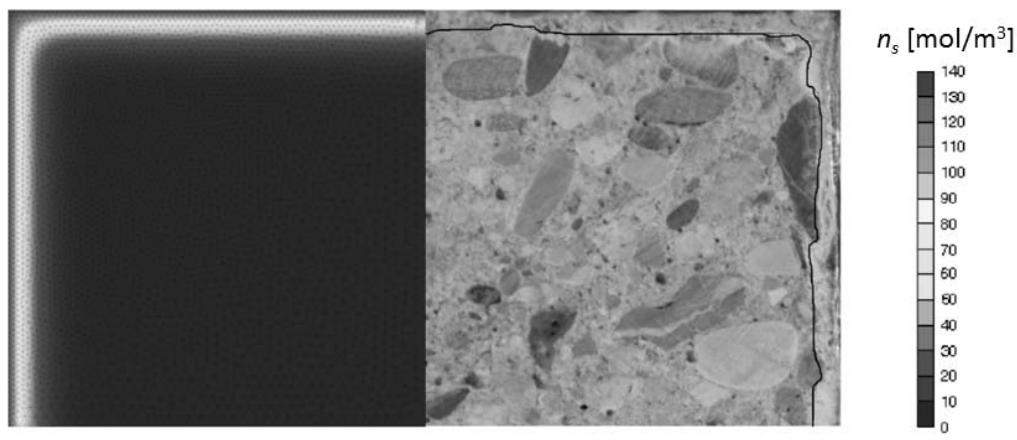

Figure 8: Penetration front of sulfate after 400 days of exposure to a $10 \% \mathrm{NaSO}_{4}$ solution: numerical simulation (left) and experimental profile (right)

The identified values of water and sulfate diffusivities and of the reaction coefficient were then used in the numerical simulation of the tests performed on cylinders in different exposure conditions. The parameters used in the chemomechanical analysis are reported in table 2 .

Figures (9) and (10) show the comparison between the model predictions and 


$$
\begin{array}{ccc}
E=30000 \mathrm{MPa} & a_{t}=0.28 & r_{1}=45 \\
\nu=0.2 & b_{t}=3.9 \mathrm{MPa} & r_{2}=5.1 \\
b=0.4 & k_{t}=17.6 \mathrm{MPa}^{2} & r_{3}=0.4 \\
M=19000 \mathrm{MPa} & D_{0 t}=0.4 & \\
& \sigma_{e} / \sigma_{0}=0.6 &
\end{array}
$$

Table 2: Material parameters used in the simulation of the experiments reported in [13]

the experimental data of longitudinal and radial strains of the cylinders stored in different conditions. The experimental points represent the mean values, with the error bars, of four specimens stored in the same conditions. The difference between the characteristic times involved in the imbibition preocess and in the chemical reaction is worthnoting: the specimens in water reach a stationary value of deformation after about three months, while the specimens affectected by the reaction after more than two years still show an increase of deformation. The proposed model is able to provide an estimate of the deformations in good agreement with the experimental observations. In particular the influence of the sulfate concentration and the effect of geometry on the difference between the longitudinal and radial deformation are well simulated.

Figure 11 shows the pattern of chemical and mechanical damage calculated by the model on a cylinder (only one quarter of the cross section is shown) after one and two years of exposure in $10 \%$ sodium sulfate solution. The chemical damage develops in a uniform way starting from the surface of the specimen in contact with the aggressive solution, while the mechanical tension damage develops below the outer layer and concentrates near the corner. This prediction is qualitatively in agreement with the experimental observation that shows circonferential cracks in correspondence of this region, see Figure 12.

As a further validation, we have also simulated the data obtained in another experimental campaign, still ongoing, on concrete prisms of $8 \mathrm{~cm} \times 8 \mathrm{~cm} \times 16 \mathrm{~cm}$ made with pure Portland cement type CEM I 52.5R. The specimens cast with 


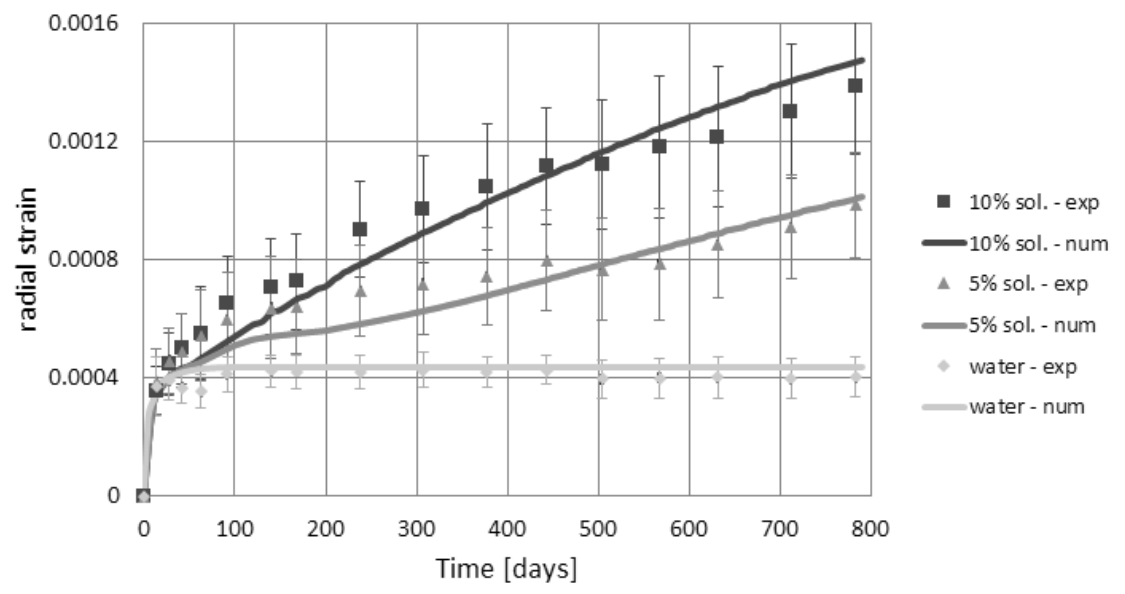

Figure 9: Radial strain of fully immersed cylinders: specimens in pure water, in $5 \% \mathrm{Na}_{2} \mathrm{SO}_{4}$ solution and in $10 \% \mathrm{Na}_{2} \mathrm{SO}_{4}$ solution. Experimental points (mean and variance on 4 specimens) and results of numerical simulations

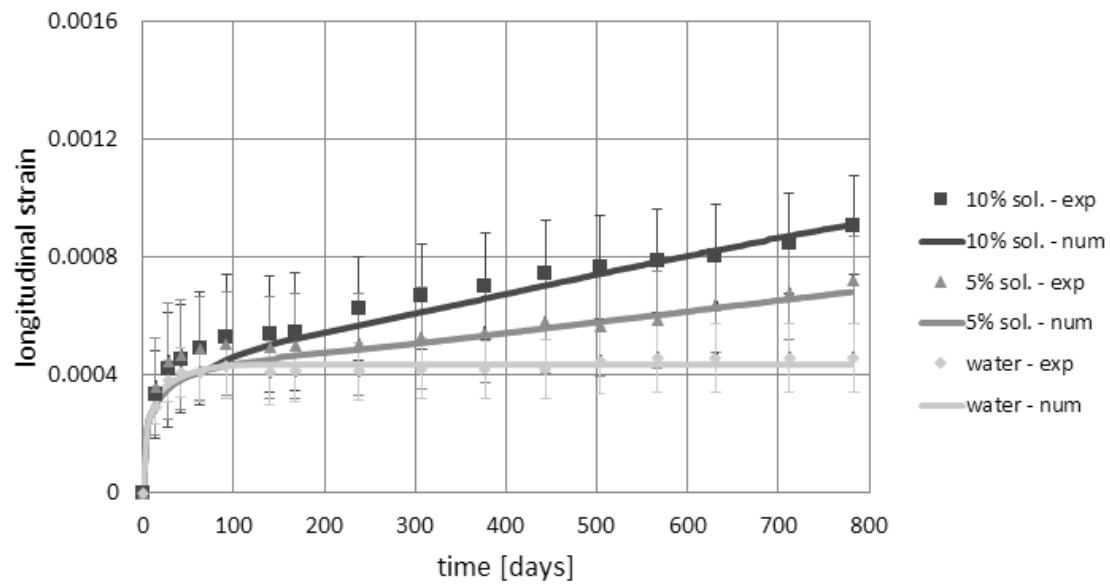

Figure 10: Longitudinal strain of fully immersed cylinders: specimens in pure water, in $5 \%$ $\mathrm{Na}_{2} \mathrm{SO}_{4}$ solution and in $10 \% \mathrm{Na}_{2} \mathrm{SO}_{4}$ solution. Experimental points (mean and variance on 4 specimens) and results of numerical simulations

this more reactive cement, after curing have been subject to different exposure conditions. Four prisms were fully immersed in pure water, four prisms were 


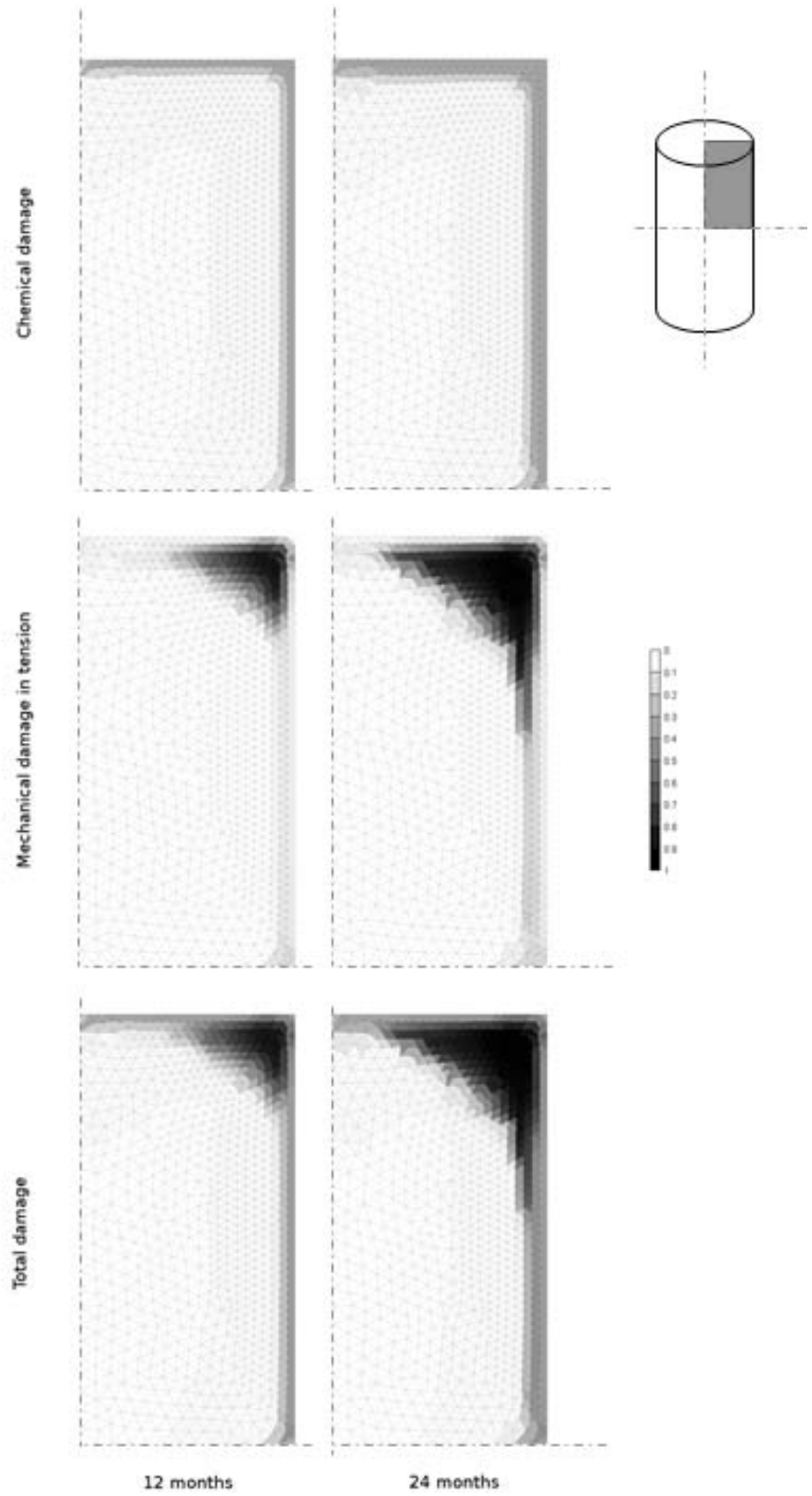

Figure 11: Chemical, mechanical (in tension) and total damage in a concrete specimen after one year and two years in a $10 \%$ sodium sulfate solution 


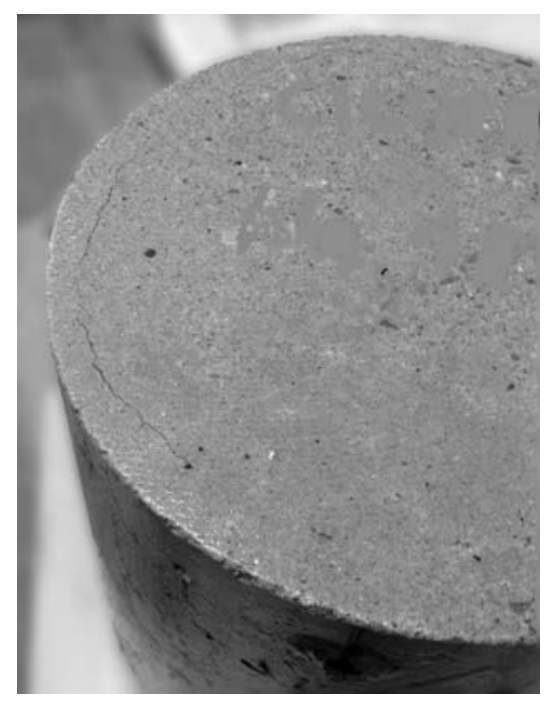

Figure 12: Crack on a specimen after two years in a $10 \%$ sodium sulfate solution

fully immersed in in sodium sulfate solution at $10 \%$, four other prisms were located in a climatic chamber at temperature $T=20^{\circ} \mathrm{C}$ and $\mathrm{RH}=90 \%$ partially immersed for half height in two different solutions: pure water and sodium sulfate solution at $10 \%$. In all cases the longitudinal and transversal deformation were measured at fixed intervals (see [20] for details).

The material parameters used in the numerical analyses are the same used to simulate the other tests. Only the value of the initial concentration of equivalent aluminates is different because of the different chemical properties of cement, namely: $n_{c}(0)=96 \mathrm{~mol} / \mathrm{m}^{3}$.

Figure 13 shows the comparison between the experimental and numerical predicted evolution of transversal and longitudinal deformation for specimens immersed in pure water, while figure 14 shows the same comparison for specimen fully immersed in $10 \%$ sodium sulfate. A good agreement is obtained in all cases.

Figure 16 shows the evolution of the longitudinal and transversal deformations for specimens partially immersed in sodium sulfate solution. In this case the transversal deformations are different in the lower part of the specimens in contact with the solutions and in the upper part of the specimens that is in air. 


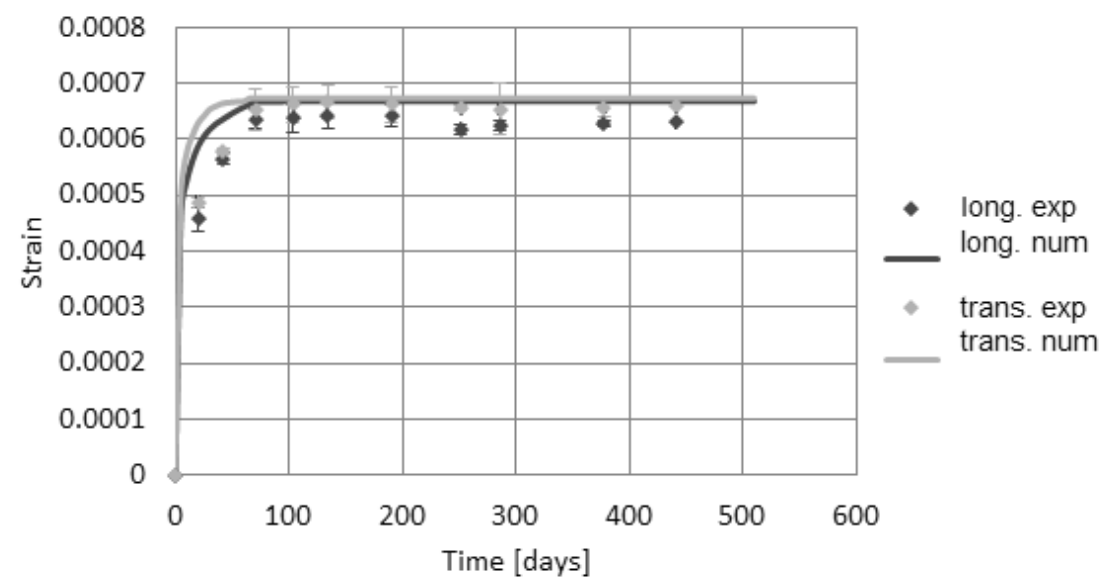

Figure 13: Radial and transversal strain of prisms immersed in pure water: experimental points (mean and variance on 4 specimens) and results of numerical simulation

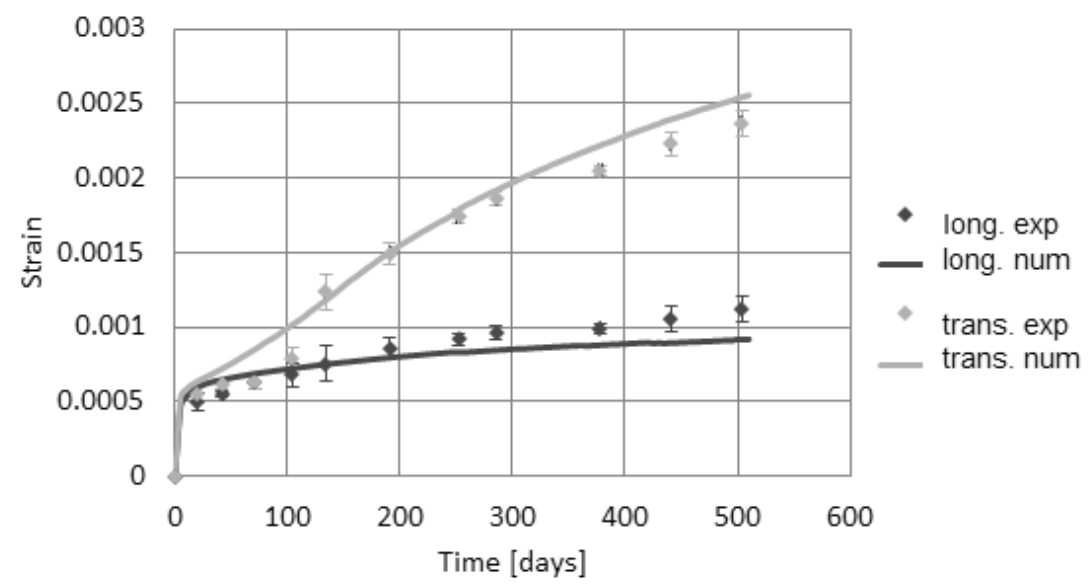

Figure 14: Radial and transversal strain of prisms immersed in $10 \% \mathrm{Na}_{2} \mathrm{SO}_{4}$ solution: experimental points (mean and variance on 4 specimens) and results of numerical simulation

The transversal deformation measured at the upper studs (number 10 and 7 in figure 15) is not appreciably influenced by the chemical reaction; on the contrary the transversal deformation measured at the lower studs (number 12 and 9 in figure 15) is significantly influenced by the reaction and it is quite similar to that 
observed in the totally immersed prisms. The longitudinal deformation exhibits a growth which remains between those of the two transversal deformations. The model correctly reproduce the observed behaviour.

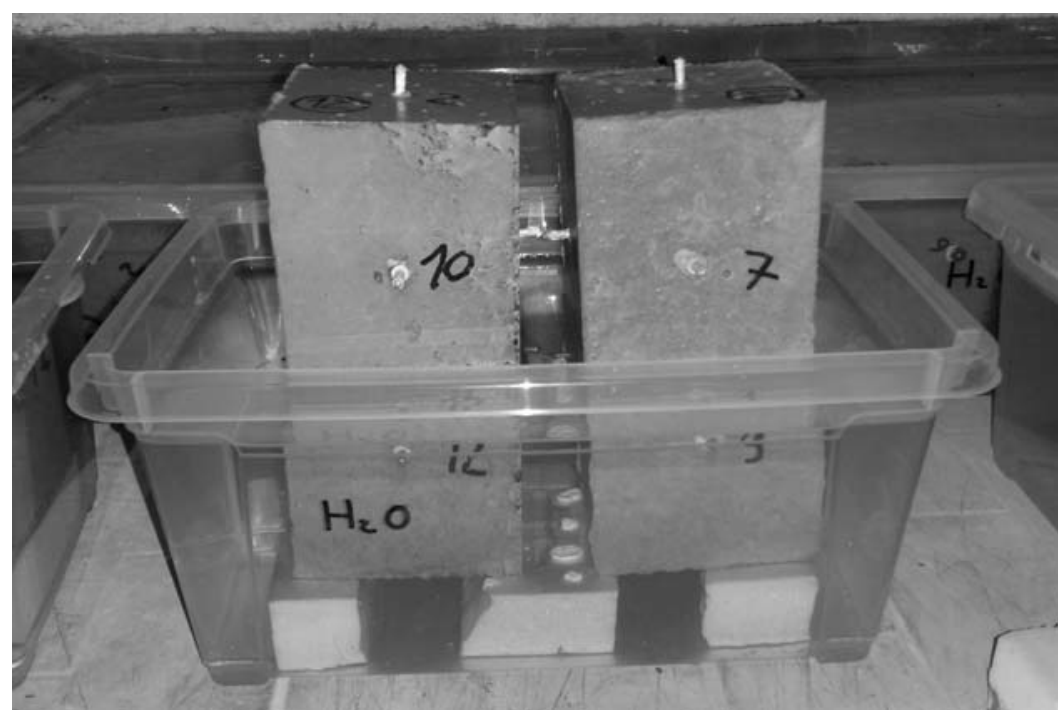

Figure 15: Prismatic specimens partially immersed; some of the studs for longitudinal and transversal measurements, labelled by numbers, are visible

\section{Tunnel subject to ESA}

As an example of sulfate attack on concrete structures, we have considered the experimental campaign reported in [1] aimed to study the long term mechanical response of tunnels in sulfate-rich soils. In that study, some reduced scale models were cast and placed in an artificial environment test system constituted by a box filled with soil and closed at the top by a steel plate, on which different mechanical loads were applied, see Figure 17. Inside the tunnel a temperature of $25^{\circ} \mathrm{C}$ and a relative humidity of $80 \%$ were fixed. A $5 \%$ sodium sulfate solution was periodically injected into the soil in order to produce the sulfate attack in the tunnel lining. The tests were performed by imposing different constant vertical loads; the displacements of the vault and of other characteristic points 


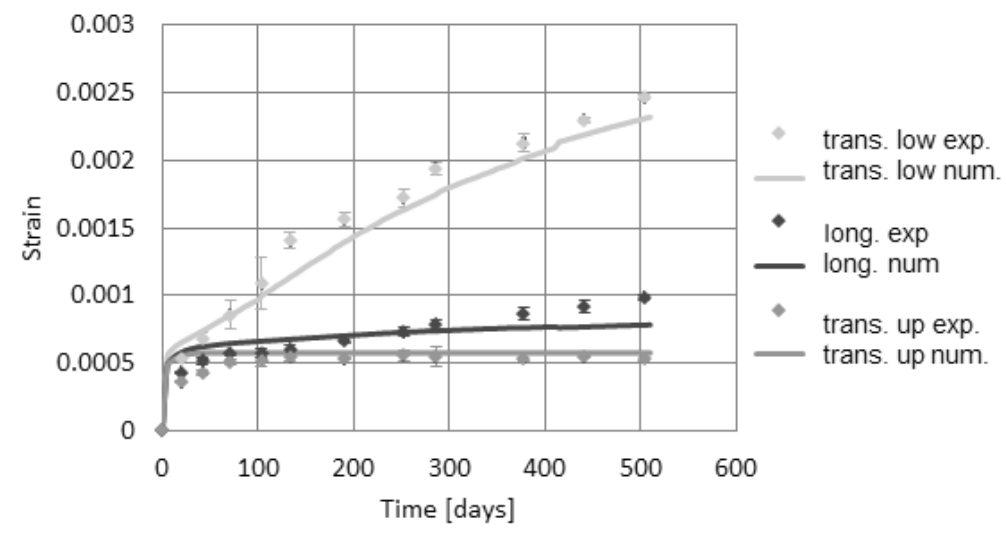

Figure 16: Prisms partially immersed in $10 \%$ sodium sulfate solution. Time evolution of longitudinal and transversal deformations: experimental mean value on 2 specimens and model simulations

of the lining were monitored during the tests.

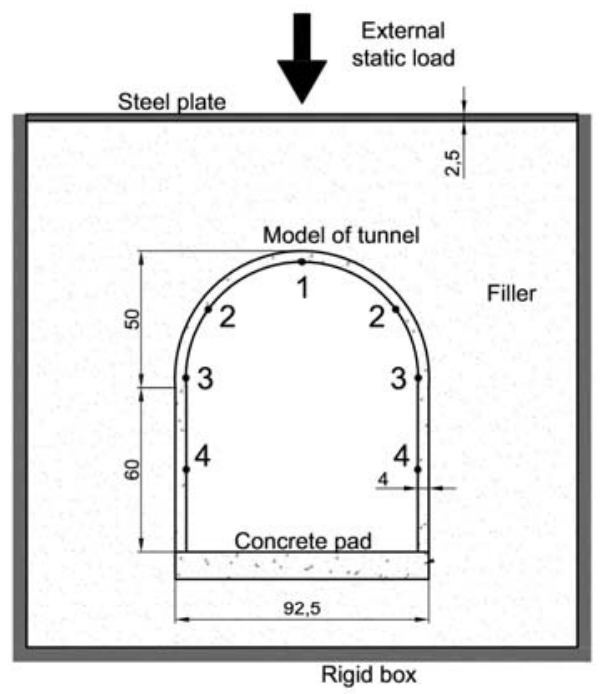

Figure 17: Geometry of the reduced scale 1:10 model of the tunnel lining structure tested in [1], (measures in [cm]).

We simulated these tests by the proposed approach, considering a plane 
strain approximation of the tunnel-soil-box system. Figure 18 shows the evolution of the vault settlement at different load levels. The symbols represent the experimental values periodically measured on the scaled model at different load levels while the solid lines are the results of the simulation. The numerical results are in good agreement with the experimental observations for values of external load $q=160 \mathrm{kN}$ and $q=190 \mathrm{kN}$. For higher loads the numerical simulation underestimates the measured displacements which are however more dispersed.

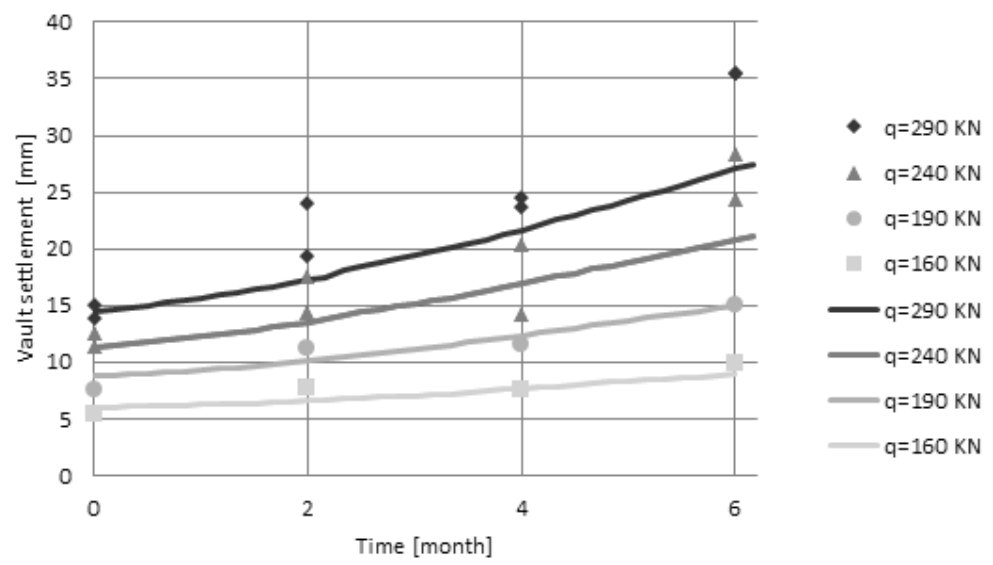

Figure 18: Comparison between experimental (symbols) and numerical (continuous lines) evolution in time of vault settlements

Figure 19 shows the comparison in terms of displacements of characteristic points of the vault and of the flanks of the tunnel after 6 months of exposure and for different load levels. A reasonably good agreement is observed.

Figure 20 displays the contour plots of the chemical, mechanical damage in tension and total damage after 2 and 6 months, for the test with $q=290 \mathrm{kN}$. It can be observed that the chemical damage progressively develops, starting from the surface in contact with the aggressive soil and then spreads through the whole thickness of the tunnel. The mechanical damage in tension concentrates at the upper part of the vault and reaches high values already after 2 months of exposure. Another highly damaged part develops at the interface between con- 


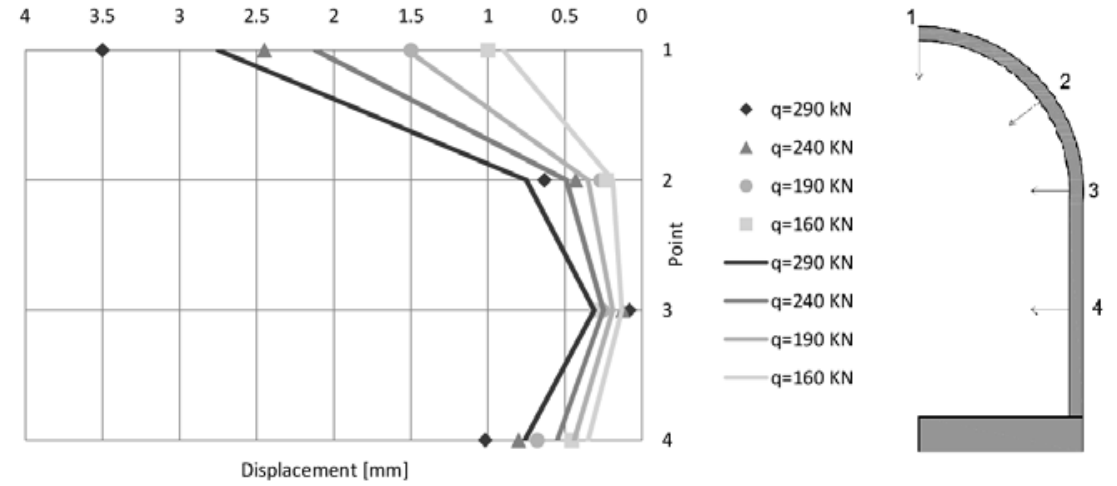

Figure 19: Comparison between the experimental and numerical displacement of the monitoring points 1,2,3 and 4 for different loads after 6 months of exposure

crete and soil at one third of the arch. The mechanical damage in compression, not shown, is very limited, less than 0.06 in the whole structure. The damage evolution is consistent with the experimental observation of cracked areas with spalling in about $10 \%$ of the lining after two months of exposure and of very large degraded zones after six months.

To test the ability of the model to assess the structural safety, we also computed the residual load bearing capacity of the tunnel, damaged due to ESA during six months of exposure under a constant load of $q=160 \mathrm{kN}$, and we compared it with the initial load bearing capacity. To that purpose, we performed two mechanical damage analyses of the tunnel subject to increasing load: one starting from the initial condition of the undamaged tunnel and the other with the initial condition of the tunnel damaged by the sulfate attack; the collapse load was identified as the maximum load of convergence in these numerical analyses. Figure 21 shows the vault settlement evolution as a function of the applied load for the tunnel without and with ESA. In the first case the limit load is $q=515 \mathrm{kN}$, while in the second case the limit load is $q=372 \mathrm{kN}$ : the damage developed due to the chemical reaction reduces the load carrying capacity of the tunnel lining of about $28 \%$. Also the reduction of stiffness is clearly visible 

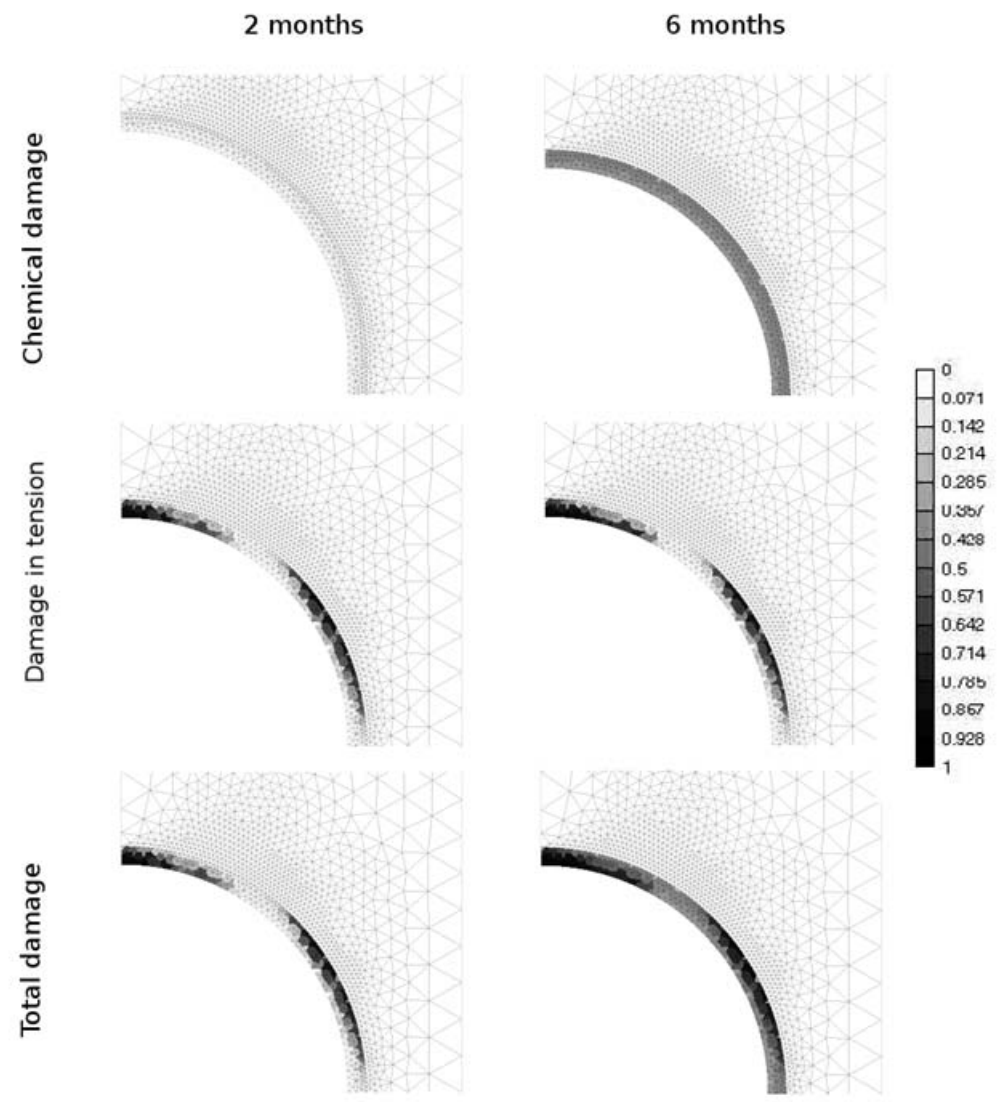

Figure 20: Patterns of chemical, mechanical damage in tension and total damage after two and six months of exposure, external load of $290 \mathrm{kN}$.

in Fig. 21.

\section{Conclusions}

The weakly coupled approach developed and implemented in this work allows for the computation of the mechanical response of concrete subject to sulfate attack also in partially saturated conditions. The multi-phase material formulation together with the definition of chemical and mechanical damage variables allows to correctly reproduce the experimental results obtained on concrete spec- 


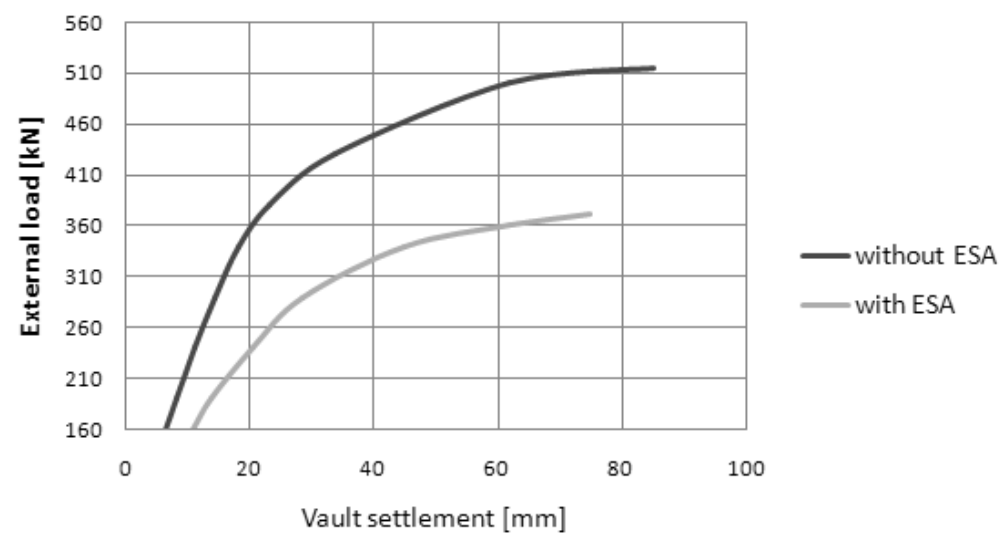

Figure 21: Vault settlement versus applied load for the tunnel without ESA and exposed to ESA for six months, with external load of $160 \mathrm{kN}$.

imens of different composition and in different environmental conditions. The different reactivity of the cement used in the mix design is directly taken into account in the model by the molar concentration of alluminates. The diffusivity parameters of the model have been directly calibrated from the experiments to reproduce the profile of salt penetration. The chemical and mechanical damage allow to describe the actual microcrack formation and the decrease of elastic properties. The weakly coupled approach followed makes the formulation simple enough to be used to effectively compute the response at the structural level. The predictive capabilities of the model have been shown on a simple structural example concerning ESA on a reduced scale tunnel lining.

\section{References}

[1] M. Lei, L. Peng, C. Shi, S. Wang, Experimental study on the damage mechanism of tunnel structure suffering from sulfate attack, Tunnelling and underground space technology 36 (2013) 5-13.

[2] M. A. Shamaa, S. Lavaud, L. Divet, G. Nahas, J. Torrenti, Coupling between mechanical and transfer properties and expansion due to def in a 
concrete of a nuclear plant, Nuclear Engineering and Design 266 (2014) $70-77$.

[3] A. Neville, The confused world of sulfate attack on concrete, Cement and Concrete Research 34.8 (2004) 1275-1296.

[4] M. Collepardi, A state-of-the-art review on delayed ettringite attack on concrete, Cement and Concrete Composites 25.4 (2003) 401-407.

[5] R. Tixier, B. Mobasher, Modeling of damage in cement-based materials subjected to external sulfate attack, i: Formulation., ASCE J. Mater. Civ. Eng 15 (2003) 305-322.

[6] M. Basista, W. Weglewski, Micromechanical modeling of sulphate corrosion in concrete: influence of ettringite forming reaction, Theoretical and Applied Mechanics 35 (2008) 29-52.

[7] E. Roziére, A. Loukili, R. E. Hachem, F. Grondin, Durability of concrete exposed to leaching and external sulphate attacks., Cement and Concrete Research 39 (2009) 1188-1198.

[8] D. Planel, J. Sercombe, P. L. Bescop, F. Adenot, J. Torrenti, Long-term performance of cement paste during combined calcium leaching sulfate attack: kinetics and size effect, Cement and Concrete Research 36.1 (2006) $137-143$

[9] S. Tracy, S. Boyd, J. Connolly, Effect of curing temperature and cement chemistry on the potential for concrete expansion due to def, PCI journal 49 (2004) 46-53.

[10] T. Wee, A. Suryavanshi, S. Wong, A. Rahman, Sulfate resistance of concrete containing mineral admixtures, ACI Materials Journal 97 (2000) 536549 .

[11] P. Akpinar, I. Casanova, A combined study of expansive and tensile strength evolution of mortars under sulfate attack: implications on durability assessment, Materiales de Construccion 60-297 (2010) 59-68. 
[12] G. Escadeillas, J. Aubert, M. Segerer, W. Prince, Some factors affecting delayed ettringite formation in heat-cured mortars, Cement and Concrete Research 37 (2007) 1445-1452.

[13] N. Cefis, C. Comi, C. Tedeschi, Swelling and degradation in concrete due to external sulfate attack, submitted.

450 [14] B. Bary, Simplified coupled chemo-mechanical modeling of cement pastes behavior subjected to combined leaching and external sulfate attack, Int J Numer Anal Meth Geomech 32 (2008) 1791-1816.

[15] A. Idiart, C. Lopez, I. Carol, Chemo-mechanical analysis of concrete cracking and degradation due to external sulfate attack: a meso-scale model., Cement and Concrete Composites 33 (2011) 411-423.

[16] M. Mainguy, O. Coussy, V. Baroghel-Bouny, Role of air pressure in drying of weakly permeable materials, Journal of engineering mechanics 127-6 (2001) 582-592.

[17] C. Comi, R. Fedele, U. Perego, A chemo-thermo-damage model for the analysis of concrete dams affected by alkali-silica reaction, Mechanics of Materials 41.3 (2009) 210-230.

[18] C. Comi, B. B. Kirchmayr, R. Pignatelli, Two-phase damage modeling of concrete affected by alkali-silica reaction under variable temperature and humidity conditions, International Journal of Solids and Structures 49 (2012) 3367-3380.

[19] F. Pesavento, D. Gawin, M. Wyrzykowski, B. Schrefler, L. Simoni, Modeling alkali silica reaction in non-isothermal, partially saturated cement based materials, Comput. Methods Appl. Mech. Engrg. 225228 (2012) 95-115.

[20] N. Cefis, Mechanical effects of sulfate attack on concrete: experimental characterization and modelling, PhD thesis, Politecnico di Milano, 2016.

[21] J. Marchand, I. Older, J. Skalny, Sulfate attack on concrete, CRC Press. 
[22] M. Santhanam, M. Cohen, J. Olek, Mechanism of sulfate attack: a fresh look: part 1: summary of experimental results, Cement and Concrete Research 32.6 (2002) 915-921.

[23] O. Coussy, Poromechanics, John Wiley Sons.

[24] F. Ulm, O. Coussy, L. Kefei, C. Larive, Thermo-chemo-mechanics of asr expansion in concrete structures, Journal of engineering mechanics 126.3 (2000) 233-242.

[25] J. Lemaitre, J. Dufailly, Damage measurements, Engineering Fracture Mechanics 28 (1987) 643-661.

[26] C. L. Bellego, G. Pijaudier-Cabot, B. Gerard, J. Dube, L. Molez, Coupled mechanical and chemical damage in calcium leached cementitious structures, J. Eng. Mech. 129(3) (2003) 333-341.

[27] R. Pignatelli, C. Comi, P. Monteiro, A coupled mechanical and chemical damage model for concrete affected by alkali-silica reaction, Cement and Concrete Research 53 (2013) 196-210.

[28] C. Comi, U. Perego, Fracture energy based bi-dissipative damage model for concrete, International Journal of Solids and Structures 38 (2001) 64276454 .

[29] C. Comi, U. Perego, Anisotropic damage model for concrete affected by alkali-aggregate reaction, International Journal of Damage Mechanics 20 (2011) 598-617.

[30] A. Campos, C. Lopez, A. Aguado, Diffusion-reaction model for the internal sulfate attack in concrete., Construction and Building Materials 102 (2016)

$495 \quad 531-540$

[31] T. Ikumi, S. Cavalaro, I. Segura, A. Aguado, Alternative methodology to consider damage and expansions in external sulfate attack modeling., Cement and Concrete Research 63 (2014) 105-116. 
[32] E. Samson, J. Marchand, K. Snyder, J. Beaudoin, Modeling ion and fluid transport in unsaturated cement systems for isothermal conditions., Cement and Concrete Research 35.1 (2005) 141-153.

[33] E. Samson, J. Marchand, Modeling the transport of ions in unsaturated cement-based materials, Computers and Structures 85 (2007) 1740-1756. 